\title{
Model-based Pedestrian Trajectory Prediction using Environmental Sensor for Mobile Robots Navigation
}

\author{
Haruka Tonoki \\ School of Science for Open and \\ Environmental Systems \\ Keio University \\ Yokohama, Japan
}

\author{
Ayanori Yorozu \\ Keio Advanced Research Centers \\ Keio University \\ Yokohama, Japan
}

\author{
Masaki Takahashi \\ Department of System Design \\ Engineering \\ Keio University \\ Yokohama, Japan
}

\begin{abstract}
Safety is the most important to the mobile robots that coexist with human. There are many studies that investigate obstacle detection and collision avoidance by predicting obstacles' trajectories several seconds into the future using mounted sensors such as cameras and laser range finder (LRF) for the safe behavior control of robots. In environments such as crossing roads where blind areas occur because of visual barriers like walls, obstacle detection might be delayed and collisions might be difficult to avoid. Using environmental sensors to detect obstacles is effective in such environments. When crossing roads, there are several passages pedestrian might move and it is difficult to depict going each passage in the same movement model. Therefore, we hypothesize that a more effective way to predict pedestrian movement is by predicting passages pedestrian might move and estimating the trajectories to the passages. We acquire pedestrian trajectory data using an environmental LRF with an extended Kalman filter (EKF) and construct pedestrian movement models using vector auto regressive (VAR) models, which pedestrian state is consisting of the position, speed and direction. Then, we test the validity of the constructed pedestrian movement models using experimental data. We narrow down the selection of a pedestrian movement model by comparing the prediction error for each path between the estimated pedestrian state using an EKF, and the predicted state using each movement model. We predict the trajectory using the selected movement model. Finally, we confirm that an appropriate path model that a pedestrian can actually move through is selected before the crossing area and that only the appropriate model is selected near the crossing area.
\end{abstract}

Keywords-Prediction of Human Movement; Service Robots; Vector Auto Regressive Models; Kalman Filter; Collision Avoidance

\section{INTRODUCTION}

Various service robots are expected to coexist with humans in real environments. Examples include guidance, communication, and assistant robots. These robots must approach a service user and avoid other humans according to the situation. Especially, for the safe behavior control of autonomous robots that coexist with humans, there are many studies that investigate obstacle detection and collision avoidance using mounted sensors such as cameras and laser range finder. For safety and collision avoidance, several methods have previously been proposed to allow autonomous robots to avoid local collisions reactively: potential field methods [1, 2], social force methods [3], dynamic window approaches [4-6], and vector field approaches [7, 8].

This study was supported by JSPS KAKENHI Grant Number 15H02890.
Furthermore, collision avoidance methods for dynamic obstacles such as pedestrian have been proposed which function by predicting obstacles' trajectories several seconds into the future and making decisions based on these predicted trajectories. At present, trajectory prediction methods are important because of the risk of a collision between obstacles and the robot when the trajectory prediction is not sufficiently accurate. With this in mind, this study focused on predicting the dynamic trajectories of pedestrians.

Several methods for predicting pedestrian trajectories assume that pedestrians move with constant speed [9-13]. This assumption may only be effective for short-term predictions because pedestrian trajectories can also change under the influence of the environment. Therefore, some pedestrian trajectory prediction methods considering pedestrian movement tendencies using pedestrian trajectory data that are observed in advance have been proposed.

Those methods predict the trajectory using the current state (pedestrian position and velocity) or the current and previous states. However, a pedestrian's trajectory changes with each step near crossing areas, for example when crossing roads at a crossing point. It may be more effective to consider the pedestrian's state several steps in the past. In this study, we constructed pedestrian movement models based on vector auto regressive (VAR) models. We approximate a pedestrian's position, speed, and direction of movement and predict their trajectory using their states several steps in the past.

Moreover, obstacles may be detected too late to avoid collisions in environments with blind areas caused by visual barriers like walls. In such environments, pedestrian movement prediction methods using environmental sensors are effective [14]. In this study, we constructed a model and predicted the pedestrian's trajectory using an environmental sensor.

It is thought that pedestrians change their direction step by step near environments where multiple passages cross (e.g., when crossing roads). There are many paths to the destination, far from the crossing area. To realize safe mobile robot navigation in such environments, we must construct each path model and predict the pedestrian trajectory, and also evaluate each predicted trajectory and select the appropriate path model for the pedestrian.

This study proposes methods that predict a pedestrian's trajectory, evaluates each predicted trajectory, and selects the 
pedestrian's approaching path using an environmental sensor. We expect that a robot can more effectively avoid pedestrians using this method than existing methods, because it reduces the number of candidate paths near the crossing area.

In concrete terms, we construct pedestrian movement models as follows. First, we acquire pedestrian trajectory data using an environmental LRF with an extended Kalman filter (EKF). Second, we construct VAR models of degree ranging from 2 to 30 for each path. Third, we compare the prediction accuracy for each degree. Then, we decide the pedestrian movement models' degree and verify the constructed models' accuracy. We narrow down the selection of a pedestrian movement model by comparing the prediction error for each path between the estimated pedestrian state using an EKF, and the predicted state using each movement model. Then, we predict the trajectory using the selected movement model. In this study, we verify the validity of the constructed pedestrian movement models using experimental data. Furthermore, we confirm that an appropriate path model that a pedestrian can actually move through is selected before the crossing area and that only the appropriate model is selected near the crossing area.

\section{RELATED WORK}

Many existing pedestrian trajectory prediction methods use the current state (e.g., pedestrian position and velocity) or the current and previous step states. Shiomi et al. proposed a method that predicts a pedestrian trajectory using the social force model [15]. Similarly, Ratsamee et al. proposed a method that predicts pedestrian trajectories using social force models, considering pedestrian's body pose, face orientation, and personal space [16]. Tamura et al. proposed a method that predicts pedestrian trajectories by storing state transition data in each $1 \mathrm{~m}^{2}$ and predicting state transitions using the current pedestrian state and the stored state transition data [17]. Tadokoro et al. proposed a method that predicts pedestrian movement by estimating movement tendencies via trial and error when a pedestrian moves in an environmental cell [18]. Noguchi et al. proposed a method that predicts pedestrian movement paths by modeling pedestrian movement between cells based on a variable length Markov model [19]. Other researchers have proposed methods that build pedestrian models using machine learning. Chung et al. used Markov decision processes [20, 21], and Ziebart et al. used a softmaximum version of Markov decision processes [22]. Callaghan et al. proposed using a Gaussian process [23] and Ellis et al. used Gaussian process regression [24].

These methods do not consider pedestrians' destinations when predicting their movement. However, several methods have been proposed that estimate destination and predict the trajectory toward that destination. Thompson et al. proposed a method that derives the transfer probability of each destination, estimates the destination using random sample consensus, and then predicts pedestrian movement using the derived transfer probability [25]. Bennewiz et al. proposed a method that estimates the destination using an expectation-maximization algorithm, and predicts the trajectory using hidden Markov models [26]. Foka et al. proposed a method that predicts a pedestrian's position at the next step using the current and previous step based on a polynomial neural network. They estimated the destination using the tangent vector of the obstacle's positions at times $t-1, t$ and the predicted position at time $t+1[27,28]$. These methods predict indoor trajectories toward destinations such as the TV and the refrigerator. However, when crossing roads where a blind area occurs because of walls, it is difficult to depict taking a right turn, going straight and then taking a left turn in the same movement model. Therefore, we hypothesize that a more effective way to predict pedestrian movement is by predicting pedestrian destination passages and estimating the paths to the passages.

\section{ACQUIREMENT OF PEDESTRIAN TRAJECTORY DATA}

We conducted an experiment to acquire pedestrian trajectory data when crossing roads using LRF (UTM-30LX, Hokuyo Automatic Co, Ltd., Japan) at the height of $0.22 \mathrm{~m}$ that is pedestrian thigh. Figure 1 shows the experimental environment and pedestrian movement direction. We observed the distance to the obstacles at each $0.050 \mathrm{~s}$ using LRF in advance. Then, we acquired the position of pedestrian on each time step and collected 157 trajectory data points in total using an EKF with position as the observation value. Table 1 shows the number of trajectory data points that we acquired. We used 142 data points for constructing models and another 15 data point for verifying the models. The process of acquiring the trajectory data is as follows.

To acquire the position data, we compared current LRF data and environmental LRF data that we acquired in advance without the presence of obstacles. Then, we acquired leg data that was different from the environmental LRF data by more than $0.10 \mathrm{~m}$. The $l(i)$-th observed position data point is

$$
\boldsymbol{P}_{l(i)}=\left(x_{l(i)}, y_{l(i)}\right)
$$

and we cluster by

$$
\left\|\boldsymbol{P}_{l(i+1)}-\boldsymbol{P}_{l(i)}\right\|>a
$$

thus $a$ is $0.10 \mathrm{~m}$. Figure 2 shows examples of data from one pair of legs, and the derived position at that time. $b$ is the width of the cluster, and the $b$ of the data from each pair of legs is less than $0.20 \mathrm{~m}$. Figure 2 (a) shows the pattern with the legs apart. In this case, we acquire the data as one pair of legs when the adjacent cluster distance $c$ is less than $1.0 \mathrm{~m}$. Figure 2 (b) shows the pattern with the two legs together. In this case, we acquire the data as one pair of legs when $b$ is more than $0.20 \mathrm{~m}$. The position of the pedestrian is at the center of the pair of legs. 


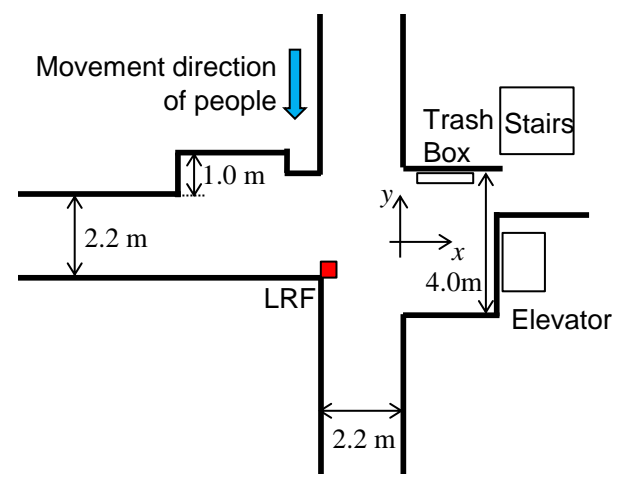

Fig. 1. Experimental environment
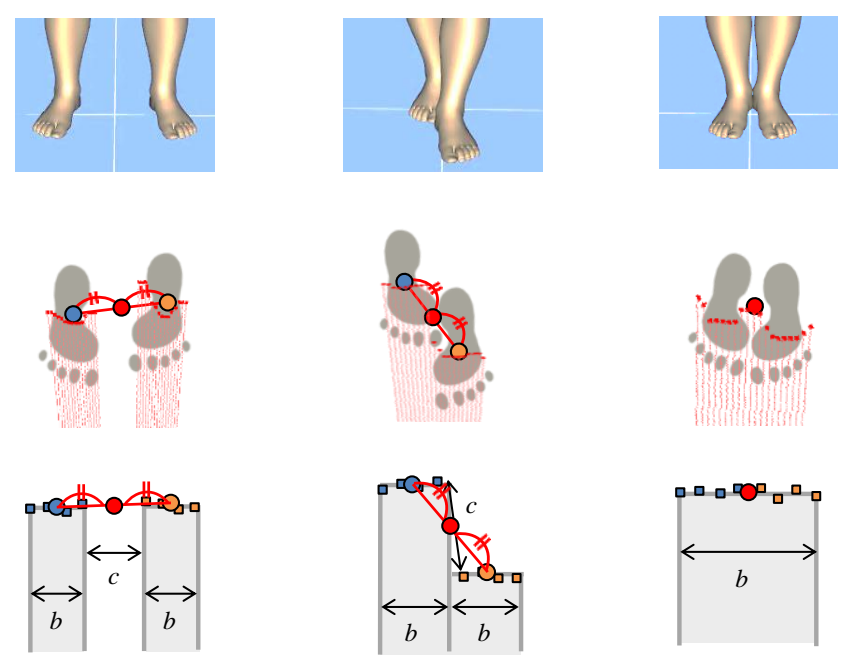

(a) Two legs apart

(b) Two legs together

Fig. 2. Leg detection

Next, we acquire the trajectory data, which consists of the position data at each time step. Acquired position data includes sensor noise stemming from the LRF accuracy, and the system noise stemming from the position acquisition process. So, we estimate the trajectory data by considering this noise using an EKF [29].

We define the pedestrian state vector $\boldsymbol{x}_{k}$ at current time step $k$ as:

$$
\boldsymbol{x}_{k}=\left[\begin{array}{llll}
x_{k} & y_{k} & \theta_{k} & v_{k}
\end{array}\right]^{T}
$$

Here, $x_{k}$ and $y_{k}$ is position, $\theta_{k}$ is movement direction, and $v_{k}$ is movement speed. The state equation and observation equation are as follows. :

$$
\begin{gathered}
\boldsymbol{x}_{k}=f\left(\boldsymbol{x}_{k-1}\right)+\boldsymbol{w}_{k-1} \\
\boldsymbol{z}_{k}=\boldsymbol{H} \boldsymbol{x}_{k}+\boldsymbol{v}_{k}
\end{gathered}
$$

thus the observation value $z_{k}$ is:

$$
\boldsymbol{z}_{k}=\left[\begin{array}{ll}
x_{k}^{L R F} & y_{k}^{L R F}
\end{array}\right]^{T}
$$

and $f\left(\boldsymbol{x}_{k}\right), \boldsymbol{H}$ is:

$$
\begin{gathered}
f\left(\boldsymbol{x}_{k}\right)=\left\{\begin{array}{l}
x_{k-1}+v_{k-1} \Delta t_{k-1} \cos \theta_{k-1} \\
y_{k-1}+v_{k-1} \Delta t_{k-1} \sin \theta_{k-1} \\
\phi_{k-1} \\
\left|v_{k-1}\right|
\end{array}\right. \\
\boldsymbol{H}=\left[\begin{array}{llll}
1 & 0 & 0 & 0 \\
0 & 1 & 0 & 0
\end{array}\right]
\end{gathered}
$$

where $\boldsymbol{w}_{k}$ is the system noise and $\boldsymbol{v}_{k}$ is the observation noise. by:

The prediction and correction steps of the EKF are given

Prediction step

$$
\begin{gathered}
\boldsymbol{x}_{k}^{-}=f\left(\boldsymbol{x}_{k-1}, 0\right) \\
\boldsymbol{P}_{k}^{-}=\boldsymbol{F}_{k} \boldsymbol{P}_{k-1} \boldsymbol{F}_{k}^{T}+\boldsymbol{Q}
\end{gathered}
$$

where $\boldsymbol{x}_{k}^{-}$is the a priori estimation value and $\boldsymbol{P}_{k}^{-}$is the error covariance; and

\section{Correction step}

$$
\begin{gathered}
\boldsymbol{S}_{k}=\boldsymbol{H P}_{k}^{-} \boldsymbol{H}^{T}+\boldsymbol{R} \\
\boldsymbol{K}_{k}=\boldsymbol{P}_{k}^{-} \boldsymbol{H}^{T} \boldsymbol{S}_{k}^{-1} \\
\boldsymbol{x}_{k}=\boldsymbol{x}_{k}^{-}+\boldsymbol{K}_{k}\left(z_{k}-\boldsymbol{H} \boldsymbol{x}_{k}^{-}\right) \\
\boldsymbol{P}_{k}=\boldsymbol{P}_{k}^{-}-\boldsymbol{K}_{k} \boldsymbol{S}_{k} \boldsymbol{K}_{k}^{T}
\end{gathered}
$$

where $\boldsymbol{K}_{k}$ is the Kalman gain that needs to be calculated, $\boldsymbol{x}_{k}$ is the a posteriori estimate value, and $\boldsymbol{P}_{k}$ is the error covariance. We define $\boldsymbol{F}_{k}, \boldsymbol{Q}, \boldsymbol{R}$ as follows:

$$
\begin{gathered}
\boldsymbol{F}_{k}=\left.\frac{\partial f(\boldsymbol{x}, 0)}{\partial \boldsymbol{x}}\right|_{\boldsymbol{x}=\boldsymbol{x}_{k}}=\left[\begin{array}{cccc}
1 & 0 & -v_{k} \Delta t_{k} \sin \theta_{k} & \Delta t_{k} \cos \theta_{k} \\
0 & 1 & v_{k} \Delta t_{k} \cos \theta_{k} & \Delta t_{k} \sin \theta_{k} \\
0 & 0 & 1 & 0 \\
0 & 0 & 0 & \operatorname{sgn}\left(v_{k}\right)
\end{array}\right] \\
\boldsymbol{Q}=\operatorname{cov}\left(\boldsymbol{w}_{k}\right)=E\left[\boldsymbol{w}_{k} \boldsymbol{w}_{k}^{T}\right]=\left[\begin{array}{cccc}
0 & 0 & 0 & 0 \\
0 & 0 & 0 & 0 \\
0 & 0 & \sigma_{\phi}^{2} & 0 \\
0 & 0 & 0 & \sigma_{v}^{2}
\end{array}\right] \\
\boldsymbol{R}=\operatorname{cov}\left(\boldsymbol{v}_{k}\right)=E\left[\boldsymbol{v}_{k} \boldsymbol{v}_{k}^{T}\right]=\left[\begin{array}{cc}
\sigma_{x}^{2} & 0 \\
0 & \sigma_{y}^{2}
\end{array}\right]
\end{gathered}
$$

where $\sigma_{\phi}$ and $\sigma_{v}$ are the variance values of the system noise, and $\sigma_{x}$ and $\sigma_{y}$ are the variance values of the 
observation noise. Considering the LRF accuracy and the amount of pedestrian movement change at each time step, we define $\boldsymbol{Q}$ and $\boldsymbol{R}$ as follows:

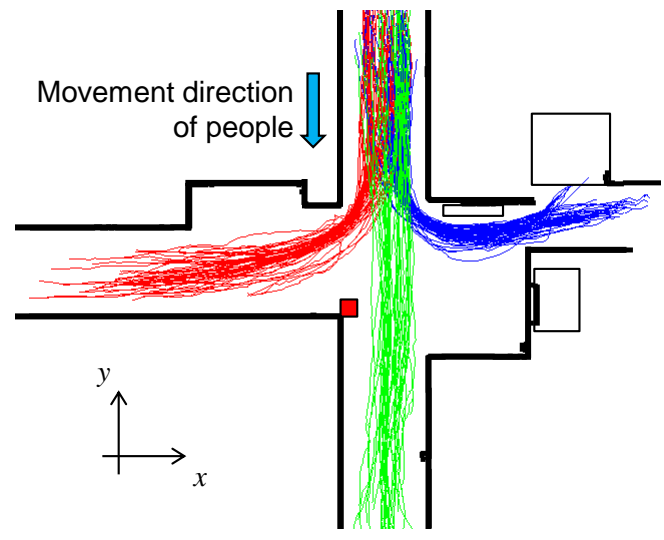

Fig. 3. Estimated human trajectories (red: right trajectory, green: straight trajectory, blue: left trajectory)

$$
\begin{gathered}
\boldsymbol{Q}=\left[\begin{array}{llcc}
0 & 0 & 0 & 0 \\
0 & 0 & 0 & 0 \\
0 & 0 & 0.0076 & 0 \\
0 & 0 & 0 & 0.000625
\end{array}\right] \\
\boldsymbol{R}=\left[\begin{array}{cc}
0.0025 & 0 \\
0 & 0.0025
\end{array}\right]
\end{gathered}
$$

where we acquire the LRF data every $0.050 \mathrm{~s}$.

Figure 3 shows the estimated pedestrian trajectory data using the EKF. In the following, the estimated pedestrian state vector $\boldsymbol{X}_{k}$ is:

$$
\boldsymbol{X}_{k}=\hat{\boldsymbol{x}}_{k}=\left[\begin{array}{llll}
\hat{x}_{k} & \hat{y}_{k} & \hat{\theta}_{k} & \hat{v}_{k}
\end{array}\right]^{T}
$$

\section{CONSTRUCtion OF PEDESTRIAN MOVEMEnT Models}

We construct pedestrian movement models using VAR models. To predict pedestrian trajectory accurately, it is necessary to use high degree models. However, more time is needed for these models to predict trajectories than for lower degree models, because they have to use more time step data. Therefore, it is necessary to construct models in which prediction error is small but degree is low. The construction of the pedestrian movement is as follows.

First, we construct each 2-30 degree VAR model. VAR models $(p)$ enable us to predict the $(k+1)$ th step in the state given the state at the $k$ th step:

$$
\hat{\boldsymbol{X}}_{k+1 \mid k}^{d}=\boldsymbol{\beta}_{0}^{d}+\sum_{i=1}^{p} \boldsymbol{\beta}_{i}^{d} \boldsymbol{X}_{k-i+1}
$$

where $d$ is a direction parameter that can be concretely right $(r)$, straight $(s)$, or left $(l)$.

Second, we derive the coefficient $\boldsymbol{\beta}^{d}$ using the maximum likelihood method for each degree to compare accuracy. The multidimensional normal distribution of $\boldsymbol{y}$ with mean $\boldsymbol{\mu}$, covariance matrix $\Sigma$ and degree $D$ is:

$$
N(\boldsymbol{y} \mid \boldsymbol{\mu}, \Sigma)=\frac{1}{\sqrt{(2 \pi)^{D}|\Sigma|}} \exp \left\{-\frac{1}{2}(\boldsymbol{y}-\boldsymbol{\mu}) \Sigma^{-1}(\boldsymbol{y}-\boldsymbol{\mu})^{T}\right\}
$$

thus $\boldsymbol{X}_{k}$ is of the $4^{\text {th }}$ degree, the likelihood function and log-likelihood function of $\boldsymbol{X}_{k}$ that have mean $\hat{\boldsymbol{X}}^{d}$ and covariance matrix $\Sigma^{d}$ are:

$$
\begin{aligned}
& L\left(\hat{\boldsymbol{X}}^{d}, \Sigma^{d}\right) \\
&= \prod_{i=1}^{n^{d}} N\left(\boldsymbol{X} \mid \boldsymbol{\mu}^{d}, \Sigma^{d}\right) \\
&= \prod_{i=1}^{n^{d}} \frac{1}{\sqrt{(2 \pi)^{4}\left|\Sigma^{d}\right|}} \exp \left\{-\frac{1}{2}\left(\boldsymbol{X}-\hat{\boldsymbol{X}}^{d}\right)\left(\Sigma^{d}\right)^{-1}\left(\boldsymbol{X}-\hat{\boldsymbol{X}}^{d}\right)^{T}\right\} \\
& \log L\left(\hat{\boldsymbol{X}}^{d}, \Sigma^{d}\right) \\
&=-2 n^{d} \log (2 \pi)-\frac{1}{2} n^{d} \log \left(\operatorname{det} \Sigma^{d}\right) \\
&-\frac{1}{2} \sum_{i=1}^{n^{d}}\left(\boldsymbol{X}_{i}-\hat{\boldsymbol{X}}_{i}^{d}\right) \Sigma^{-1}\left(\boldsymbol{X}_{i}-\hat{\boldsymbol{X}}_{i}^{d}\right)^{T}
\end{aligned}
$$

where $n^{d}$ is the number of data steps that are used to construct the VAR models, and the number for each direction is:

$$
n^{r}=2042, n^{s}=1621, n^{l}=2645
$$

We estimate VAR models' $(p)$ coefficients $\boldsymbol{\beta}_{0}^{d}, \boldsymbol{\beta}_{1}^{d}, \cdots, \boldsymbol{\beta}_{p}^{d}$, which maximize the log-likelihood function, using the maximum likelihood method.

Third, we compare the models of each degree and decide the degree of the pedestrian movement models. The center is approximately $1.1 \mathrm{~m}$ from the edge of the passage, considering the general width of the passage is $2.3 \mathrm{~m}$. Therefore, it takes approximately $2.2 \mathrm{~s}$ until the end of the avoidance procedure when the speed of the robot is assumed to be $0.50 \mathrm{~m} / \mathrm{s}$. We consider that the robot can avoid a pedestrian with enough margins by predicting the pedestrian trajectory up to $3.0 \mathrm{~s}$ in the future. Therefore, we predict the pedestrian trajectory up to $3.0 \mathrm{~s}$ in the future. Accordingly, we compare position prediction error $E_{k}^{d}$ up to $3.0 \mathrm{~s}$ in the future and decide the pedestrian movement models' degree.

$$
E_{k}^{d}=\frac{0.05}{3.0} \sum_{i=1}^{p}\left\|\boldsymbol{P}_{k-i+1}-\hat{\boldsymbol{P}}_{k-i+1 \mid k-i}^{d}\right\|
$$

where $\boldsymbol{P}_{k}$ and $\hat{\boldsymbol{P}}_{k}^{d}$ is the position vector of $\boldsymbol{X}_{k}$ and $\hat{\boldsymbol{X}}_{k}^{d}$.

The state at the $(k+j)$ th step using the data before the $k$ th step is as follows: 


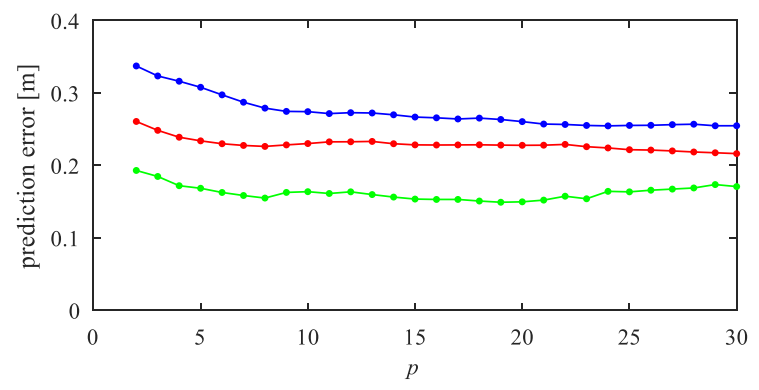

Fig. 4. Prediction error $E_{k}^{d}$ until $3.0 \mathrm{~s}$ later for each degree (red: right trajectory, green: straight trajectory, blue: left trajectory)

$$
\begin{cases}\hat{\boldsymbol{X}}_{k+j k k}^{d}=\boldsymbol{\beta}_{0}^{d}+\sum_{i=1}^{p} \boldsymbol{\beta}_{i}^{d} \boldsymbol{X}_{k+j-i} & (j=1) \\ \hat{\boldsymbol{X}}_{k+j k k}^{d}=\boldsymbol{\beta}_{0}^{d}+\sum_{i=1}^{j-1} \boldsymbol{\beta}_{i}^{d} \hat{\boldsymbol{X}}_{k+j-i}^{d}+\sum_{i=j}^{p} \boldsymbol{\beta}_{i}^{d} \boldsymbol{X}_{k+j-i} \quad(2 \leq j \leq p) \\ \hat{\boldsymbol{X}}_{k+j k}^{d}=\boldsymbol{\beta}_{0}^{d}+\sum_{i=1}^{p} \boldsymbol{\beta}_{i}^{d} \hat{\boldsymbol{X}}_{k+j-i}^{d} \quad(j>p)\end{cases}
$$

Figure 4 shows prediction error $E_{k}^{d}$ until $3.0 \mathrm{~s}$ in the future for each degree. From Fig. 4, we decide that the pedestrian movement models' degree $p=8$ because the prediction error decrease after this point is very small.

Next, we verify the appropriateness of the constructed models. The pedestrian trajectory needs to be predicted with about $0.50 \mathrm{~m}$ accuracy considering the relative sizes of pedestrians in the environment. From Fig. 4, the constructed models satisfy this prediction accuracy and enable the robot to avoid obstacles safely.

\section{PREDICTION OF PEDESTRIAN TRAJECTORY}

It is advisable to predict all trajectories that pedestrian might move for safety when crossing roads. It is difficult to predict destination passage pedestrian might move when pedestrian walks far from the crossing area. So, we assume all passages as pedestrian destinations and predict trajectories toward each passage as shown in Fig. 5. However, we can pare down the candidates near the crossing area because pedestrians change their moving direction to the destination. Therefore, we predict toward most likely passage near crossing area as shown in Fig. 6.

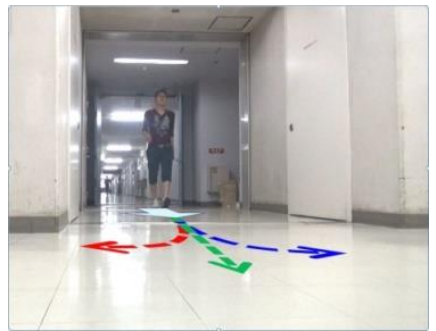

(a) Human walking situation

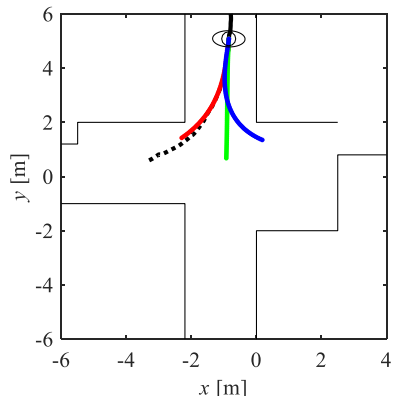

(b) Predicted trajectories
Fig. 5. Predicted trajectories when human walks far from the crossing area (black: estimated trajectory, red: predicted right trajectory, green: predicted straight trajectory, blue: predicted left trajectory)

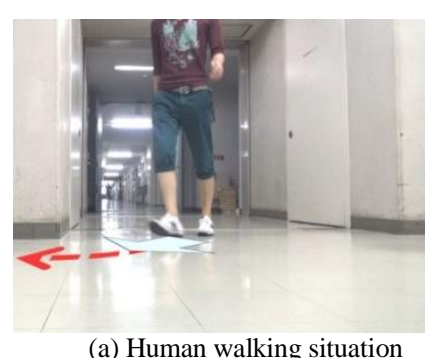

(a) Human walking situation

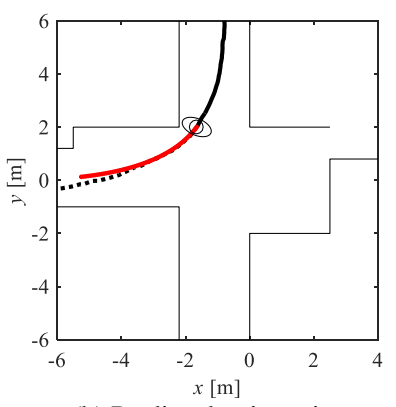

(b) Predicted trajectories
Fig. 6. Predicted trajectories when human walks near the crossing area

TABLE I. NUMBER OF EXPERIMENTAL TRAJECTORY DATA

\begin{tabular}{|c|c|c|}
\hline Subject & Model construction & Model verification \\
\hline Right trajectory data & 50 & 5 \\
\hline Straight trajectory data & 35 & 5 \\
\hline Left trajectory data & 57 & 5 \\
\hline Total & 142 & 15 \\
\hline
\end{tabular}

We predict most likely passages by comparing the average prediction error. First, we calculate average prediction error $e_{k}^{d}$ each passages and the minimum average prediction error $e_{k}^{\text {minimum }}$. Second, we select models in which $e_{k}^{d}$ is $1.0-1.5$ times of $e_{k}^{\text {minimum }} . e_{k}^{d}$ and $e_{k}^{\text {minimum }}$ are defined as follows:

$$
e_{k}^{d}=\frac{1}{p} \sum_{i=1}^{p}\left\|\boldsymbol{P}_{k-i+1}-\hat{\boldsymbol{P}}_{k-i+1 \mid k-i}^{d}\right\|
$$




$$
e_{k}^{\text {minimum }}=\min e_{k}^{d}
$$

To validate the model against the actual pedestrian trajectory, we used the verification data as shown in Table 1. Figure 7 shows the predicted and actual trajectories. Tables 2 and 3 show the $y$ coordinates at which the appropriate model was chosen.
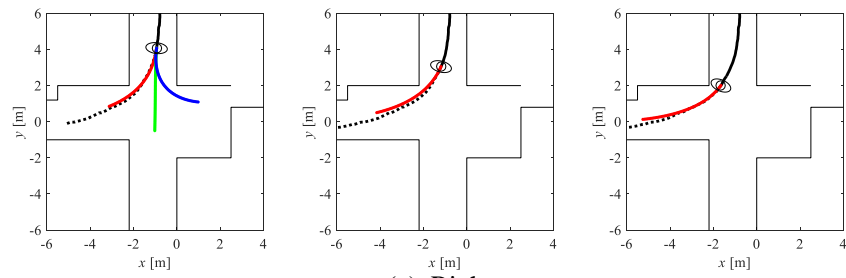

(a) Right
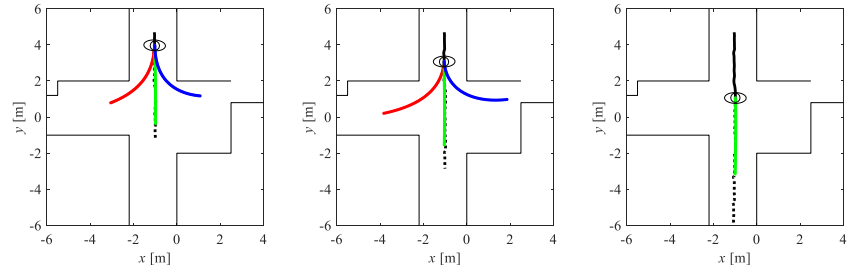

(b) Straight


(c) Left

Fig. 7. Results of human trajectory prediction

TABLE II. $\quad Y$ COORDINATE AT WHICH THE APPROPRIATE MODEL WAS CHOSEN [M]

\begin{tabular}{|l|l|l|l|}
\hline \multirow{2}{*}{ Subject } & \multicolumn{3}{|l|}{ Moving direction } \\
\cline { 2 - 4 } & Right & Straight & Left \\
\hline Mean & 5.75 & 5.22 & 5.07 \\
\hline Standard deviation & 0.80 & 0.66 & 0.87 \\
\hline
\end{tabular}

TABLE III. $\quad Y$ COORDINATE AT WHICH ONLY THE APPROPRIATE MODEL WAS CHOSEN [M]

\begin{tabular}{|l|l|l|l|}
\hline \multirow{2}{*}{ Subject } & \multicolumn{3}{|l|}{ Moving direction } \\
\cline { 2 - 4 } & Right & Straight & Left \\
\hline Mean & 1.96 & -0.59 & 1.53 \\
\hline Standard deviation & 1.03 & 0.88 & 0.34 \\
\hline
\end{tabular}

\section{DISCUSSION}

Figure 7 confirms that the number of selected models decreased and that only one appropriate model was selected near the crossing area. The selected models narrowed to only one appropriate model at $y=2.0$ when turning right (Fig. 7 (a)). However, the narrowing of the selection of models is late when heading straight and turning left (Fig. 7 (b), (c)). Moreover, in Table 2 there is no change in the point at which the appropriate model is selected when going straight or turning right or left, but the point only appreciate model selected is later when heading straight and turning left in Table 3, similar to Fig. 7.

TABLE IV. PREDICTED TRAJECTORY ERROR AT $Y=1.5$ [M]

\begin{tabular}{|c|c|c|c|}
\hline \multirow{2}{*}{ Subject } & \multicolumn{3}{|c|}{ Moving direction } \\
\cline { 2 - 4 } & Right & Straight & Left \\
\hline Mean & 0.27 & 0.10 & 0.30 \\
\hline Standard deviation & 0.03 & 0.01 & 0.03 \\
\hline
\end{tabular}

TABLE V. PREDICTED TRAJECTORY ERROR AT $Y=2.0[\mathrm{M}]$

\begin{tabular}{|c|c|c|c|}
\hline \multirow{2}{*}{ Subject } & \multicolumn{3}{|c|}{ Moving direction } \\
\cline { 2 - 4 } & Right & Straight & Left \\
\hline Mean & 0.23 & 0.20 & 0.31 \\
\hline Standard deviation & 0.03 & 0.02 & 0.03 \\
\hline
\end{tabular}

The point at which the selection of models narrows stems from the environment. The environment that we experimented with has a wide road on the right and a narrow road on the left. Moreover, most of the participants whose trajectory was acquired were students who used this environment often, and whose curvatures when turning are thought to be small when turning right and large when turning left. So, there was likely little difference in the position error when heading straight or turning left, because participants tended to begin turning further before the crossing area when turning right than when heading straight or turning left.

Tables 4 and 5 show the means and standard deviations of the prediction error $E_{k}^{d}$ at $y=1.5$ and $y=2.0$, that is, the mean points where only one appropriate model was selected when turning right and left. We confirm that the constructed models satisfy the prediction accuracy that is necessary for safe obstacle avoidance in an autonomous robot, because pedestrian trajectories need to be predicted with about $0.50 \mathrm{~m}$ accuracy considering the size of a pedestrian in the environment.

\section{CONCLUSION}

We proposed methods that predict a pedestrian's trajectory, evaluate each predicted trajectory, and select the pedestrian's approaching path using an environmental sensor, for mobile robot navigation. We believe that a robot can avoid a pedestrian with enough margins using the proposed method. Our technique predicts pedestrian trajectories by selecting likely models for environments where several passages cross, and using only one model in environments with only one passage. This method can predict trajectories of several pedestrians if combined with, for example, the potential field or social force methods, and by considering the influence of other pedestrians.

We demonstrated a method to construct pedestrian movement models based on VAR models that consist of pedestrian position, speed and direction for each passage using trajectory data that was acquired in advance by sensors in an environment where a blind area occurs to a mounted sensor on an autonomous robot when crossing roads. We also demonstrated a method of determining the degree of the model, 
such that degree is kept as low and prediction error as small as possible by comparing prediction error up to $3.0 \mathrm{~s}$ in the future. In addition, we validated the accuracy of the constructed models. Furthermore, we showed that we can predict the trajectory in which a pedestrian might move using a movement model that error is lowest between the estimated pedestrian states using an EKF and predicted the upcoming state using each model.

\section{COMPLIANCE WITH ETHICAL STANDARDS}

The authors declare that they have no conflict of interest.

\section{REFERENCES}

[1] Khatib O (1986) Real-time obstacle avoidance for manipulators and mobile robots. Int J Robot Res 5(1): 90-98

[2] Koren Y, Borenstein J (1991) Potential field methods and their inherent limitations for mo-bile robot navigation. In: Proceedings of IEEE International Conference on Robotics and Automation, pp 1398-1404

[3] Helbing D, Molnar P (1995) Social force model for pedestrian dynamics. In: Physical review E 51: 4282

[4] Fox D, Burgard W, Thrun S (1997) The dynamic window approach to collision avoidance. IEEE Robot Autom Mag 4(1): 23-33

[5] Ögren P, Leonard NE (2005) A convergent dynamic window approach to obstacle avoidance. IEEE Trans Robot 21(2): 188-195

[6] Brock O, Khatib O (1999) High-speed navigation using the global dynamic window approach. In: Proceedings of IEEE International Conference on Robotics and Automation, pp 341-346

[7] Borenstein J, Koren Y (1991) The vector field histogram-fast obstacle avoidance for mobile robots. IEEE Trans Robot Autom 7(3): 278-288

[8] Borenstein J, Koren Y (1990) Real-time obstacle avoidance for fast mobile robots in cluttered environments. In: Proceedings of IEEE International Conference on Robotics and Automation, pp 572-577

[9] Ohki T, Nagatani K, Yoshida K (2010) Collision avoidance method for mobile robot considering motion and personal spaces of evacuees. In: Proceedings of IEEE/RSJ International Conference on Intelligent Robots and Systems (IROS), pp 1819-1824

[10] Granata C, Bidaud P (2012) A framework for the design of person following behaviors for social mobile robots. In: Proceedings of IEEE/RSJ International Conference on Intelligent Robots and Systems (IROS), pp 4652-4659

[11] Tsubouchi T, Arimoto S (1994) Behavior of a mobile robot navigated by an "iterated forecast and planning" scheme in the presence of multiple moving obstacles. In: Proceedings of IEEE International Conference on Robotics and Automation, pp 2470-2475

[12] Belkhouche F (2009) Reactive path planning in a dynamic environment. IEEE Trans Robot 25(4): 902-911

[13] Pacchierotti E, Christensen HI, Jensfelt P (2006) Evaluation of passing distance for social robots. In: Proceedings of IEEE International Symposium on Robot and Human Interactive Communication (ROMAN), pp 315-320

[14] Batalin MA, Sukhatme GS, Hattig M (2004) Mobile robot navigation using a sensor network. In: Proceedings of IEEE International Conference on Robotics and Automation, pp 636-641
[15] Shiomi M, Zanlungo F, Hayashi K, Kanda T (2014) Towards a socially acceptable collision avoidance for a mobile robot navigating among pedestrians using a pedestrian model. Int J Soc Robot 6(3): 443-455

[16] Ratsamee P, Mae Y, Ohara K, Takubo T, Arai T (2013) Human-robot collision avoidance using a modified social force model with body pose and face orientation. Int J Soc Humanoid Robot 10(1): 1350008

[17] Tamura Y, Hamasaki S, Yamashita A, Asama H (2013) Collision avoidance of mobile robot based on prediction of human movement according to environments. Transactions of the Japan Society of Mechanical Engineers 79(799): 617-628 (in Japanese)

[18] Tadokoro S, Hayashi M, Manabe Y, Nakami Y, Takamori T (1995) Motion planner of mo-bile robots which avoid moving human obstacles on the basis of stochastic prediction. In: Proceedings of IEEE International Conference on Intelligent Systems for the 21st Century, pp 3286-3291

[19] Noguchi H, Yamada T, Mori T, Sato T (2012) Mobile robot path planning using human prediction model based on massive trajectories. In: Proceedings of IEEE International Conference on Networked Sensing Systems (INSS), pp 1-7

[20] Chung SY, Huang HP (2010) A mobile robot that understands pedestrian spatial behaviors. In: Proceedings of IEEE/RSJ International Conference on Intelligent Robots and Systems (IROS), pp 5861-5866

[21] Chung SY, Huang HP (2012) Incremental learning of human social behaviors with feature-based spatial effects. In: Proceedings of IEEE/RSJ International Conference on Intelligent Robots and Systems (IROS), pp 2417-2422

[22] Ziebart BD, Ratliff N, Gallagher G, Mertz C, Peterson K, Bagnell JA, Hebert M, Dey AK, Srinivasa S (2009) Planning-based prediction for pedestrians. In: Proceedings of IEEE/RSJ International Conference on Intelligent Robots and Systems (IROS), pp 3931-3936

[23] Callaghan ST, Singh SP, Alempijevic A, Ramos FT (2011) Learning navigational maps by observing human motion patterns. In: Proceedings of IEEE International Conference on Robotics and Automation, pp 4333-4340

[24] Ellis D, Sommerlade E, Reid I (2009) Modelling pedestrian trajectory patterns with gaussian processes. In: Proceedings of 12th IEEE International Conference on Computer Vision Workshops, pp 12291234

[25] Thompson S, Horiuchi T, Kagami S (2009) A probabilistic model of human motion and navigation intent for mobile robot path planning. In: Proceedings of IEEE International Conference on Autonomous Robots and Agents (ICARA), pp 663-668

[26] Bennewitz M, Burgard W, Cielniak G, Thrun S (2005) Learning motion patterns of people for compliant robot motion. Int J Robot Res 24(1): $31-48$

[27] Foka AF, Trahanias PE (2002) Predictive autonomous robot navigation. In: Proceedings of IEEE/RSJ International Conference on Intelligent Robots and Systems (IROS), pp 490-495

[28] Foka AF, Trahanias PE (2010) Probabilistic autonomous robot navigation in dynamic environments with human motion prediction. Int J Soc Robot 2(1): 79-94

[29] Bellotto N, Hu H (2010) Computationally efficient solutions for tracking people with a mo-bile robot: an experimental evaluation of Bayesian filters. Autonomous Robots 28(4): 425-438 